\title{
Breast cancer with rare metastatic manifestation
}

Francesca Magnoni*,1, Clementina Di Tonno², Giuseppe Accardo1, Mariarosaria Calvello ${ }^{3}$, Giovanni Corso ${ }^{1,4}$, Virgilio Sacchini ${ }^{1,4}$, Viviana Galimberti ${ }^{1}$ \& Paolo Veronesi ${ }^{1,4}$

${ }^{1}$ IEO, European Institute of Oncology, IRCCS, Division of Breast Cancer Surgery, Milan, Italy

${ }^{2}$ IEO, European Institute of Oncology, IRCCS, Division of Pathology, Milan, Italy

${ }^{3}$ IEO, European Institute of Oncology, IRCCS, Division of Cancer Prevention \& Genetics, Milan, Italy

${ }^{4}$ Faculty of Medicine, University of Milan, Milan, Italy

*Author for correspondence: Tel.: + 39 0294371092; francesca.magnoni@ieo.it

\section{"In this dissertation of metastatic appearance of pancreatic adenocarcinoma on the breast, we \\ illustrate the vague traits of its clinical behavior, underlying how pathological support and correlation with a patient's genetic and oncological history are mandatory for a proper diagnosis, in order to avoid unnecessary surgical procedure and to provide an adequate clinical management."}

First draft submitted: 7 May 2019; Accepted for publication: 16 May 2019; Published online: 24 July 2019

Keywords: breast metastasis $\bullet$ immunohistochemistry $\bullet$ PALB2 $\bullet$ pancreatic adenocarcinoma

Metastases to the breast represent approximately $2 \%$ of all mammary malignancies and are characterized by poor prognosis. The most common metastatic lesion to involve the breast is metastasis from a contralateral mammary cancer [1-3]. Excluding hematologic malignancies, the rate of nonmammary metastases drops to well below $1 \%$ [1,2].

In literature, a wide variety of primitive solid cancers from many different sources has been reported: the most common include gynecologic site, in particular ovarian, skin, specially melanoma, lung and gastrointestinal tract $[4-8]$.

Breast metastases from pancreatic adenocarcinoma are very rare, as documented in literature. Domanski et al. reported one case of breast metastasis as the first manifestation of a poorly differentiated pancreatic adenocarcinoma [9]. In 2001, Georgiannos et al. described one case of breast metastasis from pancreatic adenocarcinoma diagnosed in one woman in 1955, diagnosis resulted in an autopsy series, obtained from the Surgical and Postmortem Archives of the Royal London Hospital Histopathology Department [1]. Moreover, in a recent study, De Lair $e t$ al. described two cases of pancreatic metastasis among 85 patients with metastatic lesions to the breast [2].

Breast metastasis from pancreatic adenocarcinoma represents an interesting issue, due to its heterogeneous and indistinct clinical features. Immunohistochemical analysis, genetic assessment and oncological history of patient evaluated in a multidisciplinary context, are all precious tools to set up a proper diagnosis and plan a relevant management of this infrequent clinical condition.

In this dissertation of metastatic appearance of pancreatic adenocarcinoma on the breast, we illustrate the vague traits of its clinical behavior, underlying how pathological support and correlation with a patient's genetic and oncological history are mandatory for a proper diagnosis, in order to avoid unnecessary surgical procedure and to provide an adequate clinical management.

Extramammary metastases to the breast are often associated with the presence of other metastases from the primary cancer [10]. They can be detected as palpable breast masses, or on screening mammography with the integration of breast ultrasound. Breast metastases may be also incidentally identified through computed tomography (CT) imaging, or positron emission tomography (PET), commonly performed in staging and follow-up of primary extramammary neoplastic diseases. Nevertheless, their clinical and radiological features do not significantly differ from those of a primary breast malignancy, so the first appearance of a metastasis to the breast may be misinterpreted as primary breast cancer. Indeed, they have heterogeneous and not distinctive imaging hallmarks [11,12]. In this 
perspective, fine needle aspiration biopsy (FNAB) plays a key role in correct diagnosis of this disease, and decisive is the support of immunohistochemistry in differentiating primary from metastatic breast lesion [4].

\section{Pathology}

Specific immunohistochemical markers have a significant part in the study of such tumors.

Cytokeratins phenotyping, in particular CK7 and CK20, which are well-characterized immune-histochemical markers, are helpful in determining the site of origin of an unknown source's metastatic tumor [13]. Both cytokeratins may be associated to pancreatic primitiveness, in line with literature reports: in their review study, Tot et al. reported $87 \%$ of CK7 positivity and $62 \%$ of CK20 positivity in metastatic pancreatic adenocarcinoma [14]. Moreover, Morini et al. underlined the significance of CK20 expression-independent factors related to prognosis for pancreatic-biliary cancer [15].

Furthermore, CDX2 immunohistochemistry is a considerable used tool even if - in literature - its role in metastatic pancreatic adenocarcinoma is not clearly known. CDX2 is a typical homeobox domain containing transcription factor involved in intestinal development by regulating the proliferation and differentiation of intestinal cells [16]. Werling et al. and Barbareschi et al. both reported CDX2 expression in a percentage of pancreatobiliary adenocarcinoma [17,18], even if, as specified by Xiao et al., with an expression pattern heterogeneous and less strong then in colon cancer [19]. These studies also underlined the correlation between CDX2 expression and worst survival in pancreatic adenocarcinoma.

Finally, a further valuable immunomarker in differentiating primary breast cancer from other carcinomas is immunohistochemical analysis of Gata-3 expression: it is widely considered a marker for breast carcinoma, in particular, associated to luminal subtype, presenting high specificity and sensitivity in ER-positive and Her2positive subtypes [20,21]. The lack of its expression should significantly strengthen the diagnostic hypothesis of nonmammary primitiveness.

\section{Germline PALB2 mutations}

A patient's genetic history may represent an accessory mean in diagnosis of pancreatic metastasis to the breast. Pancreatic adenocarcinoma, according to several studies, is the third most common cancer associated with $B R C A$ mutations, in particular BRCA2 [22]. Moreover, recently the relevance of identification of germline PALB2 mutations in pancreatic cancer has been more deeply investigated in the literature [23-26].

The role of germline PALB2 mutations in non-BRCA1/BRCA2 hereditary breast cancer is widely studied. PALB2 is a recently discovered moderate-risk breast cancer susceptibility gene [27,28]. The PALB2 gene product functions as a tumor suppressor and interacts closely with both $B R C A 1$ and $B R C A 2$ during double-strand DNA repair. PALB2 acts as a bond protein between $B R C A 1$ and $B R C A 2$ to form a ' $B R C A$ complex' that is implicated in homologous recombination [28-30].

In breast cancer patients, germline mutations of PALB2 are rare, varying from 0.1 to $2.7 \%$ depending on the population [28,31]. In a recent paper, Li et al. reported a relationship between PALB2 expression and a specific breast cancer clinical behavior: high-level expression of PALB2 at immunohistochemical analysis in the breast cancer tissue results in significant correlation with poor prognosis, rapid progression to advanced stage and aggressive phenotype [29].

PALB2 germline mutations have also recently been shown to be associated with an increased risk of familial pancreatic cancer [27], and appear to be more prevalent in those patients with a family history of pancreatic cancer. It seemed that PALB2 mutations occur with a prevalence of $2.1 \%$ in a population of $B R C A 1 / 2$-negative breast cancer patients specifically selected for a personal and/or family history of pancreatic cancer [27].

Although the majority of pancreatic adenocarcinoma cases appear to be sporadic, about $5-10 \%$ occurs in inherited cancer predisposition syndrome [25]. Shindo et al. showed that the prevalence of germline mutations of PALB2 is about $0.2 \%$ in patients affected by apparently sporadic pancreatic cancer [26], proving that PALB2 is one of the main genes involved in pancreatic cancer predisposition, while the most common mutated gene is $B R C A 2$, whose protein product is a binding partner for PALB2 protein [26].

Further studies are necessary to define the prevalence of PALB2 mutations and their clinical utility for those individuals affected with both breast and/or pancreatic cancers.

The advantage of pancreatic cancer screening in patients at increased risk of developing pancreatic cancer, such as those who carry a BRCA2 or PALB2 mutation, is uncertain [27]. 


\section{Conclusion}

Breast metastases from extramammary tumors are infrequent, particularly the nonhematologic types. They affect preferentially the fifth-sixth decades, with primary malignancies originating from skin, lung, ovary and gastrointestinal tract [2]. In particular, breast metastases from pancreatic adenocarcinoma are quite uncommon; a few cases are reported in the literature $[1,2,9]$. Pancreatic ductal adenocarcinoma most commonly metastasizes to lymph nodes, liver, lung and peritoneal cavity, while rarely to the bone, brain, myocardium, umbilicus and esophagus [32].

Radiological instruments, as reported in literature, do not offer correct and diriment means to resolve it $[6,33]$, because of the absence of pathognomonic imaging signs of breast metastasis. Decisive in its management are pathological acquisitions, supported by immunohistochemistry and contextualized in the oncological history of the patient. Genetic counseling could be helpful to investigate the possibility of a specific germline mutation, making further elements useful in breast metastasis management, even if its clinical significance is still an object of scientific studies [34].

A careful clinical history is essential, with special attention to the simultaneous or previous diagnosis of extramammary malignancy, combined to a radiological and pathological examination. In particular, contribution of immunohistochemistry is decisive to differentiate these lesions from primary breast cancers. All these aspects, discussed in a multidisciplinary assessment, are all crucial to ensure accurate diagnosis, avoid useless surgery and define correct systemic treatment.

\section{Financial \& competing interests disclosure}

The authors have no relevant affiliations or financial involvement with any organization or entity with a financial interest in or financial conflict with the subject matter or materials discussed in the manuscript. This includes employment, consultancies, honoraria, stock ownership or options, expert testimony, grants or patents received or pending, or royalties.

No writing assistance was utilized in the production of this manuscript.

\section{References}

1. Georgiannos SN, Chin J, Goode AW, Sheaff M. Secondary neoplasms of the breast: a survey of the 20th century. Cancer 92(9), 2259-2266 (2001).

2. De Lair DF, Corben AD, Catalano JP, Vallejo CE, Brogi E, Tan LK. Non-mammary metastases to the breast and axilla: a study of 85 cases. Mod. Pathol. 26, 343-349 (2012).

3. Hajdu SI, Urban JA. Cancers metastatic to the breast. Cancer 29(6), 1691-1696 (1972).

4. Koch A, Richter-Marot A, Wissler MP, Baratte A, Mathelin C. Mammary metastasis of extramammary cancers: current knowledge and diagnostic difficulties. Gynecol. Obstet. Fertil. 41, 653-659 (2013).

5. McIntosh IH, Hooper AA, Millis RR, Greening WP. Metastatic carcinoma within the breast. Clin. Oncol. 2, 393-401 (1976).

6. Bitencourt AGV, Gama RRM, Graziano L et al. Breast metastases from extramammary malignancies: multimodality imaging aspects. Br. J. Radiol. 90, 20170197 (2017).

7. Williams SA, Ehlers RA 2nd, Hunt KK et al. Metastases to the breast from nonbreast solid neoplasms: presentation and determinants of survival. Cancer 110(4), 731-737 (2007).

8. Akcay MN. Metastatic disease in the breast. Breast 11, 526-528 (2002).

9. Domanski HA, Mas-Morillas A. Breast metastasis from pancreatic and ovarian carcinoma. Diagn. Cytopathol. 21, 154-155 (1999).

10. Tsung Swei H. Metastasis of colon cancer to the breast. Case Rep. Oncol. 10, 77-80 (2017).

11. Sippo DA, Kulkarni K, Carlo PD et al. Metastatic disease to the breast from extramammary malignancies: a multimodality pictorial review. Curr. Probl. Diagn. Radiol. 45(3), 225-232 (2016).

12. Kalli S, Lanfranchi M, Alexander A, Makim S, Freer PE. Spectrum of extramammary malignant neoplasms in the breast with radiologic-pathologic correlation. Curr. Probl. Diagn. Radiol. 45(6), 392-401 (2016).

13. Tot T. Adenocarcinomas metastatic to the liver: the value of cytokeratins 20 and 7 in the search for unknown primary tumors. Cancer 85, 171-174 (1999).

14. Tot T. Cytokeratins 20 and 7 as biomarkers: usefulness in discriminating primary from metastatic adenocarcinoma. Eur. J. Cancer 38 , $758-763$ (2002).

15. Morini S, Perrone G, Borzomati D et al. Carcinoma of the ampulla of Vater. Morphological and immunophenotypical classification predicts overall survival. Pancreas 42, 60-66 (2013).

16. Li MK, Folpe AL. CDX-2, a new marker for adenocarcinoma of gastrointestinal origin. Anat. Pathol. 11, 101-105 (2004).

17. Werling RW, Yaziji H, Bacchi CE, Gown AM. CDX2, a highly sensitive and specific marker of adenocarcinomas of intestinal origin. Am. J. Surg. Pathol. 27(3), 303-310 (2013). 
18. Barbareschi M, Murer B, Colby TV et al. CDX-2 homeobox gene expression is a reliable marker of colorectal adenocarcinoma metastases to the lungs. Am. J. Surg. Pathol. 27(2), 141-149 (2003).

19. Xiao W, Hong H, Awadallah A, Zhou L, Xin W. Utilization of CDX2 expression in diagnosing pancreatic ductal adenocarcinoma and predicting prognosis. PLoS ONE 9(1), e86853 (2014).

20. Davis DG, Siddiqui MT, Oprea-Ilies G et al. GATA-3 and FOXA1 expression is useful to differentiate breast carcinoma from other carcinomas. Hum. Pathol. 47, 26-31 (2016).

21. Ni YB, Tsang JYS, Shao MM et al. GATA-3 is superior to GCDFP-15 and mammaglobin to identify primary and metastatic breast cancer. Breast Cancer Res. Treat. 169(1), 25-32 (2018).

22. Ghiorzo P, Pensotti V, Fornarini G et al. Contribution of germline mutations in the BRCA and PALB2 genes to pancreatic cancer in Italy. Fam. Cancer 11, 41-47 (2012).

23. Slater EP, Langer P, Niemczyk E et al. PALB2 mutations in European familial pancreatic cancer families. Clin. Genet. 78(5), 490-494 (2010).

24. Stadler ZK, Salo-Mullen E, Sabbaghian N et al. Germline PALB2 mutation analysis in breast-pancreas cancer families. J. Med. Genet. 48, 523-525 (2011).

25. Salo-Mullen EE, O’Reilly E, Kelsen D et al. Identification of germline genetic mutations in pancreatic cancer patients. Cancer 121(24), 4382-4388 (2015).

26. Shindo $\mathrm{K}, \mathrm{Yu} \mathrm{J}$, Suenaga $\mathrm{M}$ et al. Deleterious germline mutations in patients with apparently sporadic pancreatic adenocarcinoma. J. Clin. Oncol. 35(30), 3382-3390 (2017).

27. Hofstatter EW, Domchek SM, Miron A et al. PALB2 mutations in familial breast and pancreatic cancer. Fam. Cancer 10, 225-231 (2011).

28. Xia B, Sheng Q, Nakanishi K et al. Control of BRCA2 cellular and clinical functions by a nuclear partner, PALB2. Mol. Cell 22, 719-729 (2006).

29. Li J, Li M, Chen P, Ba Q. High expression of PALB2 predicts poor prognosis in patients with advanced breast cancer. FEBS Open Bio 8, 56-63 (2017).

30. Zhang F, Ma J, Wu J et al. PALB2 links BRCA1 and BRCA2 in the DNA-damage response. Curr. Biol. 19, 524-529 (2009).

31. Southey MC, Teo ZL, Winship I. PALB2 and breast cancer: ready for clinical translation! Appl. Clin. Genet. 6, 43-52 (2013).

32. Rosati LM, Kummerlowe MN, Poling J et al. A rare case of esophageal metastasis from pancreatic ductal adenocarcinoma: a case report and literature review. Oncotarget 8(59), 100942-100950 (2017).

33. Bartella L, Kaye J, Perry NM et al. Metastases to the breast revisited: radiological-histopathological correlation. Clin. Radiol. 58, 524-531 (2003).

34. Harinck F, Kluijt I, Van Mil SE et al. Routine testing for PALB2 mutations in familial pancreatic cancer families and breast cancer families with pancreatic cancer is not indicated. Eur. J. Hum. Genet. 20(5), 577-579 (2012). 